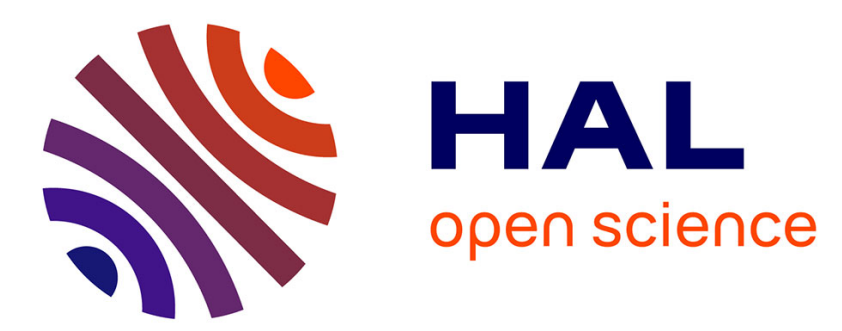

\title{
Comparison of multiple flux quantum transition with the corresponding I-U-characteristic using Nb-point contacts
}

\author{
G. Voss, A. Hausen
}

\section{To cite this version:}

G. Voss, A. Hausen. Comparison of multiple flux quantum transition with the corresponding IU-characteristic using Nb-point contacts. Revue de Physique Appliquée, 1980, 15 (2), pp.323-330. 10.1051/rphysap:01980001502032300 . jpa-00244732

HAL Id: jpa-00244732

https://hal.science/jpa-00244732

Submitted on 1 Jan 1980

HAL is a multi-disciplinary open access archive for the deposit and dissemination of scientific research documents, whether they are published or not. The documents may come from teaching and research institutions in France or abroad, or from public or private research centers.
L'archive ouverte pluridisciplinaire HAL, est destinée au dépôt et à la diffusion de documents scientifiques de niveau recherche, publiés ou non, émanant des établissements d'enseignement et de recherche français ou étrangers, des laboratoires publics ou privés. 


\title{
Comparison of multiple flux quantum transition with the corresponding $I$ - $U$-characteristic using Nb-point contacts
}

\author{
G. Voss and A. Hausen $(*)$ \\ II. Physikalisches Institut der Universität zu Köln, D 5000 Köln 41, R.F.A. \\ (Reçu le 13 juin 1979, révisé le 8 octobre 1979, accepté le 15 octobre 1979)
}

\begin{abstract}
Résumé. - Pour des contacts à pointe S-S il a été contrôlé quantitativement, seulement d'après les résultats des caractéristiques $I-U$, si les propriétés de la transition multiple des quanta du flux dans une bague sc faiblement couplée, peuvent être prédites. Ceci a été entrepris d'après le modèle de circuit shuntée par une résistance (RSJ) avec une relation courant-phase sinusoïdale modifiée. Il a été construit un appareil d'essai afin de mesurer aussi bien les caractéristiques $I-U$ que la pénétration du flux des mêmes contacts. La concordance quantitative entre la prédiction du modèle (RSJ) et les résultats de l'expérience est étonnament bonne.
\end{abstract}

\begin{abstract}
For Superconductor-Superconductor (S-S) point contacts (Nb) a quantitative check is done whether the properties of flux quantum transition into a weakly connected superconducting loop can be predicted from the shape of the corresponding I-U-characteristic. The background is taken from the Resistively Shunted Junction (RSJ)-model using a modified sinusoidal Current-Phase-Relation (CPR). An experimental set-up was built to measure the $I-U$-characteristic and the flux penetration at the same point contact. The quantitative agreement between the predictions of the RSJ-model and the results of the experiment is surprisingly good.
\end{abstract}

1. Introduction. - The Josephson-like $I-U$-characteristics of Superconductor-Superconductor (S-S) point contacts at temperatures well below $T_{\mathrm{c}}$ usually show well established hysteresis and critical currents in the $\mathrm{mA}$-region.

One can use S-S point contacts to form weakly connected superconducting (sc) loops. These loops show the well known effect of discontinuous flux penetration. The smallest possible flux to penetrate into the loop is the flux quantum $\phi_{0}$, and much effort has been made to study the single flux quantum transition [1].

Contacts exhibiting $I-U$-characteristics without hysteresis as well as single flux quantum transitions in a sc loop are still of significant interest in order to obtain quantum devices with high resolution [2].

The case of multiple flux quantum transitions is possible as well and was first theoretically considered by Kurkijärvi [3]. The first experiments showing multiple flux quantum transitions were done by [4] and [5].

Each of the two phenomena : hysteretic $I-U$ characteristic and multiple flux quantum transitions, can be described by the phenomenological Resistively Shunted Junction (RSJ)-model [6]. But there is no

(*) Present adress : RWW-Filter, Ratingen, West-Germany. quantitative check yet whether the two phenomena can be understood consistently. Of special interest is the question of how well the properties of the multiple flux quantum transition (shape of the voltage pulse, amount of the penetrated flux and the penetration time) can be predicted simply by using parameters taken from the $I-U$-characteristic of the same Josephson-contact.

Although there is the competing self-heating model [7] which describes the $I-U$-characteristic nicely we decided to use the R:SJ-model. We did this because only the RSJ-model gives a simple possibility to compare results from the $I-U$-characteristic and multiple flux quantum transitions.

For that an experimental set-up was built to measure both the I-U-characteristic and the multiple flux quantum transition using the same contact.

In discussing the experiments it will be seen that, even in cases of nonsinusoidal Current-Phase-Relation (CPR), the quasistatic RSJ-model leads to quantitatively consistent results.

2. Experiments. - An experimental arrangement was set up which allowed us to measure the $I-U$ characteristic as well as the flux penetration using the same point contact (Fig. 1). First with open switch the $I-U$-characteristic is recorded using a standard 
(a) sc switch open
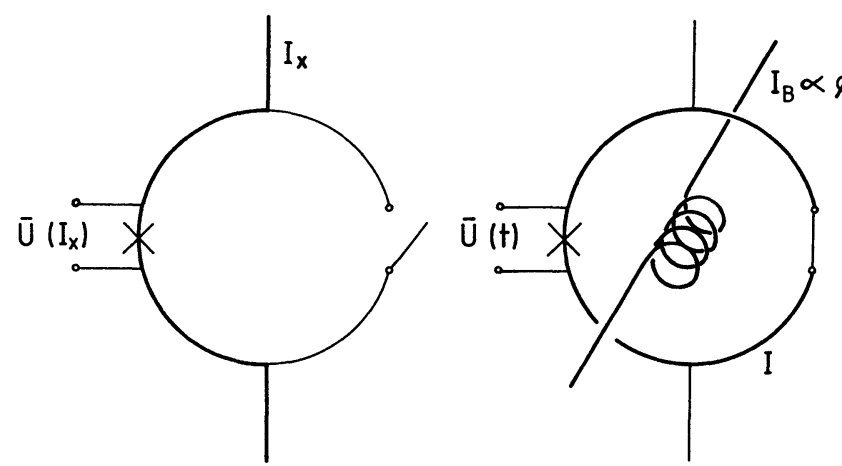

Fig. 1. - (a) Arrangement to measure the I-U-characteristic. (b) Arrangement to measure the flux penetration.

four-point-method. By closing the switch a weakly connected sc loop arises. Then an external flux is applied and the change in trapped flux is observed by measuring the voltage $U(t)=\dot{\phi}(t)$ across the contact.

The sample arrangement (Fig. 2) consists of two cylindrical Nb-disks $(1,2)$ with appropriate holes for the point contact $(7,8)$, the sc switch $(9,10)$ and the coil (5) to generate an external flux $\phi_{x}$. The $\mathrm{Nb}$-disks are insulated from each other by a mylar-foil (4).

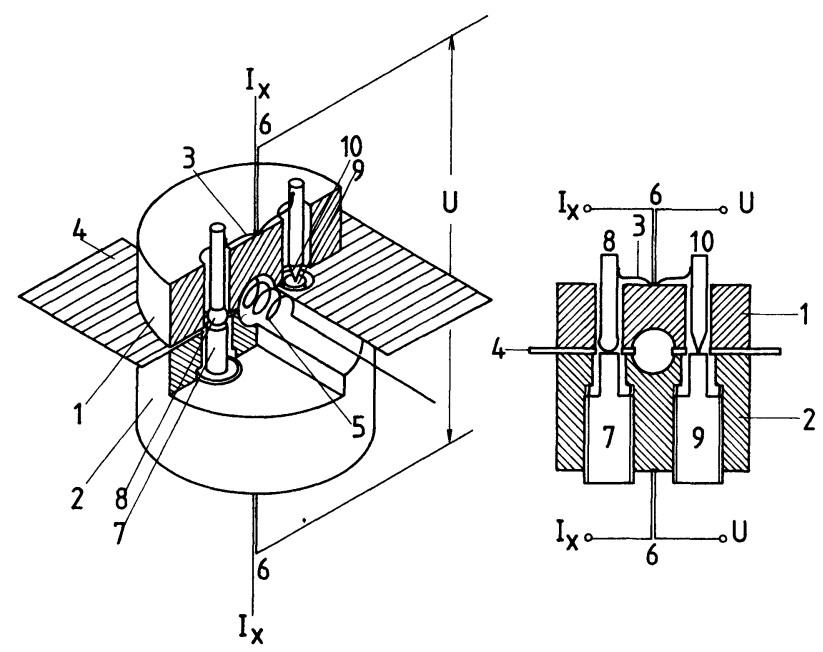

Fig. 2. - Sample arrangement and sc switch. 1, $2: \mathrm{Nb}$-disks (diameter $\simeq 25 \mathrm{~mm}) .3: \mathrm{Nb}$-wire $($ diameter $\simeq 100 \mu \mathrm{m}) .4:$ Mylarfoil (100 $\mu \mathrm{m}$ thick). 5 : Coil to generate the external flux $\phi_{x} .6$ :Current- and voltage-leads.

$\left.\begin{array}{r}7: \text { Nb-rod } \\ 8: \text { Nb-ball }\end{array}\right\}$ point contact. $\left.\begin{array}{r}9 \\ 10: \text { In-rod } \\ \text { Nb-tip }\end{array}\right\}$ sc switch.

The point contact consists of a Nb-rod (7) with a thread, which is screwed into the disk (2) tightly and a Nb-ball (8) as a tip, which is connected to the disk (1) by a Nb-wire (3). This wire is spot-welded to the tip at one end and clamped to the disk (1) by a screw at the other end. The sc switch has the same design, but the tip (10) is so sharp that one easily gets a metallic short to the counterelectrode (9) made of indium.

This arrangement leads to a high inductance $(\approx 20 \mathrm{nH})$ compared with typical values $\left(\approx 10^{-2} \mathrm{nH}\right)$ of SQUID-circuits.

The whole sample mounting is surrounded by a mu-metal shield with a damping factor near $10^{3}$. All measurements were carried out in helium bath at $1.5 \mathrm{~K}$.

Typical $I-U$-characteristics corresponding to two different states $\alpha, \beta$ of the contact are presented in figure $3 \alpha, \beta$. They are characterized by well established hysteresis $\left(I_{1}<I_{\mathrm{c}}\right)$, high critical current $I_{\mathrm{c}}$ (some $\mathrm{mA}$ ) and nonvanishing $I_{2}$ (excess current) (see Fig. 3).

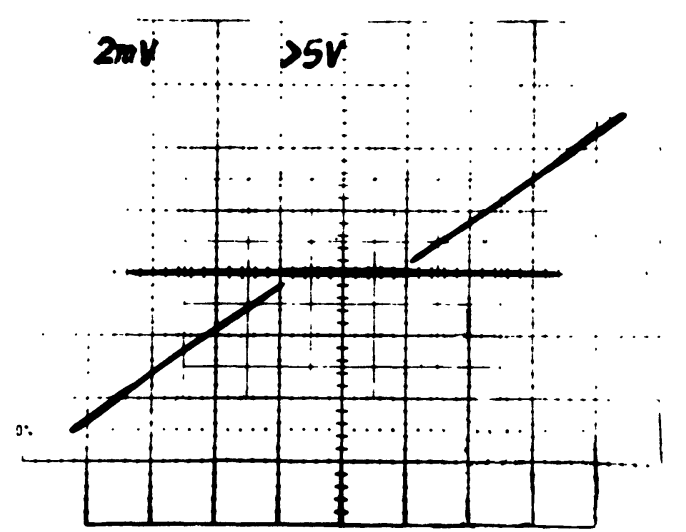

$(\alpha)$

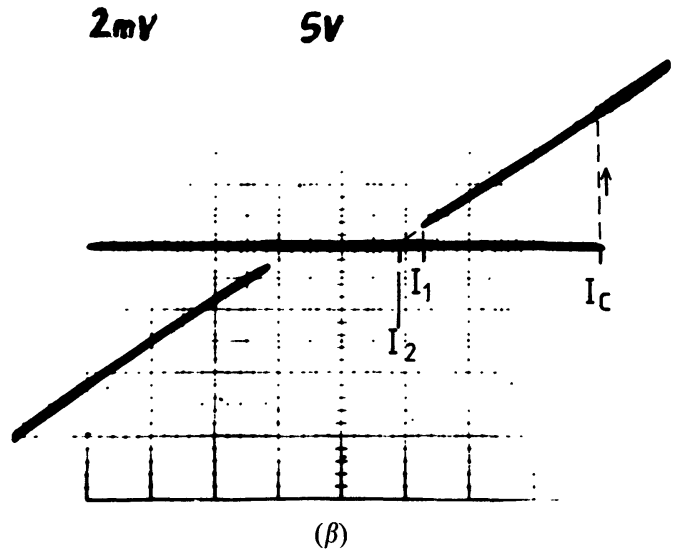

Fig. 3. - I- $U$-characteristics. $(\alpha) X$-axis : Current ; $0.74 \mathrm{~mA} /$ div $Y$-axis : Voltage; $2.0 \mathrm{mV} /$ div. $I_{\mathrm{c}}=2.6 \mathrm{~mA} . \quad I_{2}=0.6 \mathrm{~mA}$. $I_{1}=0.8 \mathrm{~mA} . R=1.7 \Omega$. ( $(\beta) X$-axis : Current $; 0.5 \mathrm{~mA} /$ div. $Y$-axis : Voltage; $1.0 \mathrm{mV} /$ div. $I_{\mathrm{c}}=2.0 \mathrm{~mA} . I_{2}=0.4 \mathrm{~mA} . I_{1}=0.6 \mathrm{~mA}$. $R=1.4 \Omega$.

In order to measure the flux penetration into the weakly connected sc loop a triangular shaped current $I_{\mathrm{B}}$ at a frequency of $20 \mathrm{kHz}$ is fed to the coil (5) (see Fig. 2). The generated external flux $\phi_{x}$ varies nearly linearly in time at a repetition-frequency $v=20 \mathrm{kHz}$. The variation of the voltage across the contact in time is recorded by a $100 \mathrm{MHz}$-scope. Typical voltage pulses due to flux penetration are 
presented in figure $4 \alpha, \beta$. For the two states $\alpha, \beta$ of the contact the corresponding $I-U$-characteristics are shown in figure $3 \alpha, \beta$ respectively.

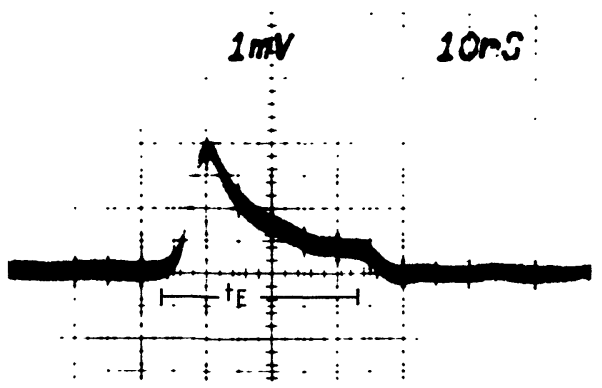

$(x)$

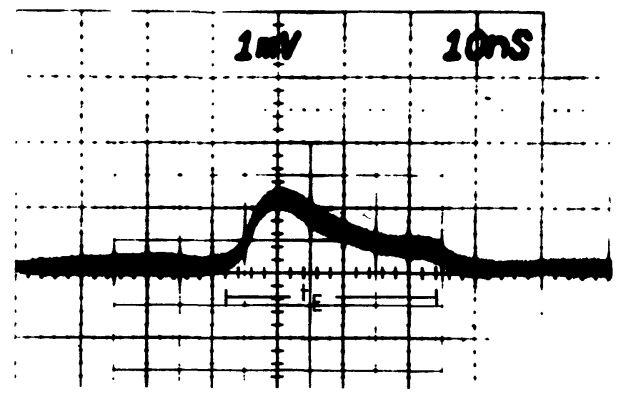

( $\beta)$

Fig. 4. - Voltage pulses due to flux penetration. $X$-axis : Time; $10 \mathrm{~ns} /$ div. $Y$-axis : Voltage; $1 \mathrm{mV} /$ div. $(\alpha) t_{\mathrm{E}} \simeq 30 \mathrm{~ns},(\beta) t_{\mathrm{E}} \simeq 32 \mathrm{~ns}$.

During the measurement the I-U-characteristic, the pulse, the $I-U$-characteristic and again the pulse are recorded in order to verify that the properties of the contact were not changed using the sc switch. Only those runs are taken into account for which the two I-U-characteristics and the two pulses are the same within the accuracy of the measurement.

3. I-U-characteristic and flux penetration in the RSJ-model. - The simplest phenomenological description of a Josephson-contact is given by the Resistively Shunted Junction (RSJ)-model [6]. The total current $I$ through the contact is composed of three parts :

$$
I=C \dot{U}+\frac{1}{R} U+I_{\mathrm{s}}
$$

where the supercurrent $I_{\mathrm{s}}$ is a function of the phase difference $\delta$ across the contact, $C$ is the capacitance and $R$ is the resistance of the weak link.

The dependence

$$
I_{\mathrm{s}} \equiv I_{\mathrm{s}}(\delta)=I_{\mathrm{c}} \cdot f(\delta)
$$

is called Current Phase Relation (CPR). The CPR is a $2 \pi$-periodic function and can therefore be written as a Fourier-series. The nonvanishing coefficients of the Fourier-expansion determine the strength of the coupling [9].
Sandwich tunnel junctions usually show weak coupling (sinusoidal CPR), whereas microbridges and point contacts usually show strong coupling (nonsinusoidal CPR). In case of point contacts, as used in this work, the surface properties of the two electrodes are affected by pressing them against each other. When a Josephson-contact is obtained it is assumed that the natural $\mathrm{Nb}$-oxide layers (1 500$2500 \AA$ thick, see ref. [10]) are destroyed so that microbridges appear or they get so thin that Josephsontunnelling arises. However, the exact geometry of the contact region is not known. Therefore there have been only a few theoretical discussions - for instance [11] - of the CPR of point contacts.

For microbridges, however, relevant CPRs are calculated on the basis of a simple model in $[12,13]$ and [14]. It turns out that in general the CPR is nonsinusoidal.

One only gets a sinusoidal CPR in case of weak coupling under the additional condition that the geometric length $l$ of the bridge is short compared with the coherence length $\xi_{\mathrm{B}}$ in the bridge. If $l$ is long compared with $2 \xi_{\mathrm{B}}$, the CPR shows reentrantbehaviour (see Fig. $5 b$ ).

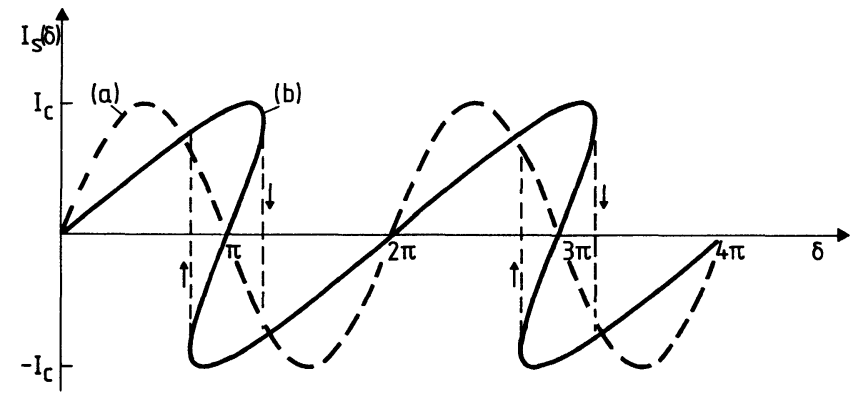

Fig. 5. - (a) Sinusoidal CPR. (b) Reentrant CPR.

The Nb-point contacts used in this work show high critical currents $I_{\mathrm{c}}$ (some $\mathrm{mA}$ ) as do sandwich tunnel junctions. But because of the small effective contact area in point contacts, there is a very high critical current density which cannot be understood by (Josephson)-electron pair tunnelling. Therefore we will treat point contacts as microbridges.

Because of the high critical current density and the assumption that the length of the bridge is of the same order of magnitude as the thickness of the $\mathrm{Nb}$-oxide layers, that means $l>2 \xi_{\mathrm{B}}$, it results from the calculations in $[12,13]$ that reentrant-behaviour of the CPR is to be expected for our contacts.

In the following considerations a reentrant CPR is approximated by a triangular CPR. This is a good approximation according to figure $5 b$ for increasing and decreasing $\delta$ respectively. Furthermore the triangular CPR can be approximated to first order by

$$
\left.\begin{array}{ll}
I_{\mathrm{s}}(\delta)=I_{2}+I_{\mathrm{c}}^{+} \sin \left(\delta-\delta_{0}\right) ; & \delta \text { increasing } \\
I_{\mathrm{s}}(\delta)=-I_{2}+I_{\mathrm{c}}^{+} \sin \left(\delta+\delta_{0}\right) ; & \delta \text { decreasing }
\end{array}\right\}
$$


as shown in figure 6 for $I_{2}=I_{\mathrm{c}}^{+}=I_{\mathrm{c}} / 2 ; \delta_{0}=\pi$ and $\delta$ increasing.

This approximation allows to transfer the results from literature for sinusoidal CPR to reentrant CPRs.

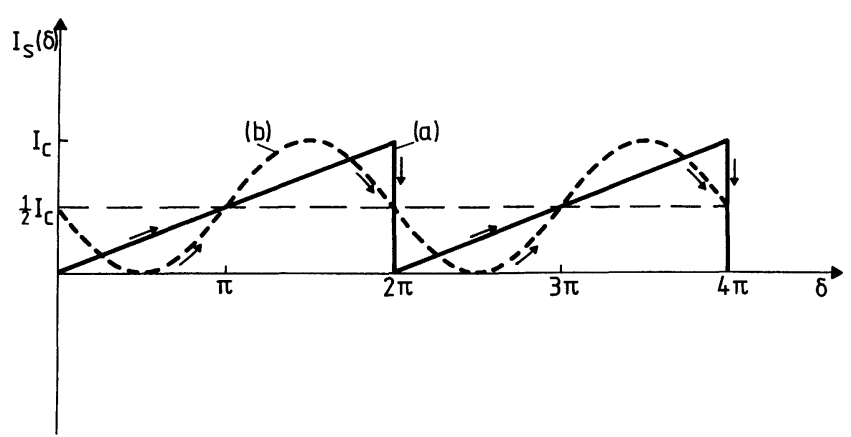

Fig. 6. - (a) Triangular CPR. (b) Modified sinusoidal CPR.

3.1 I-U-CHARACTERISTIC. - Using the Josephsonrelation

$$
\dot{\delta}=\frac{2 \pi}{\phi_{0}} U
$$

eqs. (1), (2), and the condition $I=I_{x}$ one gets a differential equation for $\delta(t)$ :

with

$$
\ddot{\delta}+\frac{1}{\tau} \dot{\delta}+\omega_{\mathrm{c}}^{2} f(\delta)=\omega_{\mathrm{c}}^{2} \frac{I_{x}}{I_{\mathrm{c}}}
$$

$$
\tau=R C \quad \text { and } \quad \omega_{\mathrm{c}}^{2}=\frac{2 \pi}{\phi_{0}} \cdot \frac{I_{\mathrm{c}}}{C}
$$

Experimentally it is not possible to measure the exact variation of the voltage in time

$$
U(t)=\frac{\phi_{0}}{2 \pi} \dot{\delta}(t),
$$

but one can record the time average $\bar{U}$ in quasistatic approximation

$$
\bar{U}=\lim _{t \rightarrow \infty} \frac{\delta(t)-\delta(0)}{t}
$$

vs. $I_{x}$ and yields the $I-U$-characteristic of the contact.

As shown above reentrant-behaviour is typical for the contacts used in this work and (3) is taken as a first approximation for the calculation.

We get

with

$$
\ddot{\delta}^{+}+\frac{1}{\tau} \dot{\delta}^{+}+\left(\omega_{\mathrm{c}}^{+}\right)^{2} \sin \delta^{+}=\left(\omega_{\mathrm{c}}^{+}\right)^{2} \frac{I_{x}^{+}}{I_{\mathrm{c}}^{+}}
$$

$$
\begin{aligned}
\delta^{+} & =\delta-\delta_{0} \\
\left(\omega_{\mathrm{c}}^{+}\right)^{2} & =\frac{2 \pi}{\phi_{0}} \cdot \frac{I_{\mathrm{c}}^{+}}{C} \\
I_{\mathrm{c}}^{+} & =I_{\mathrm{c}}-I_{2} \\
I^{+} & =I_{1}-I_{2} .
\end{aligned}
$$

This is the same type of differential equation as used in the calculations [6]. Depending on the value of

$$
\omega_{\mathrm{c}}^{+} \tau=\sqrt{\frac{2 \pi}{\phi_{0}}} R \sqrt{C I_{\mathrm{c}}^{+}}
$$

hysteresis occurs. Figure $7 b$ shows such a calculated $I-U$-characteristic for

$$
I_{2}=I_{\mathrm{c}}^{+}=I_{\mathrm{c}} / 2 ; \quad \delta_{0}=\pi,
$$

that means for

$$
I_{\mathrm{s}}(\delta)=I_{\mathrm{c}} / 2(1-\sin \delta), \quad \delta \text { increasing } .
$$

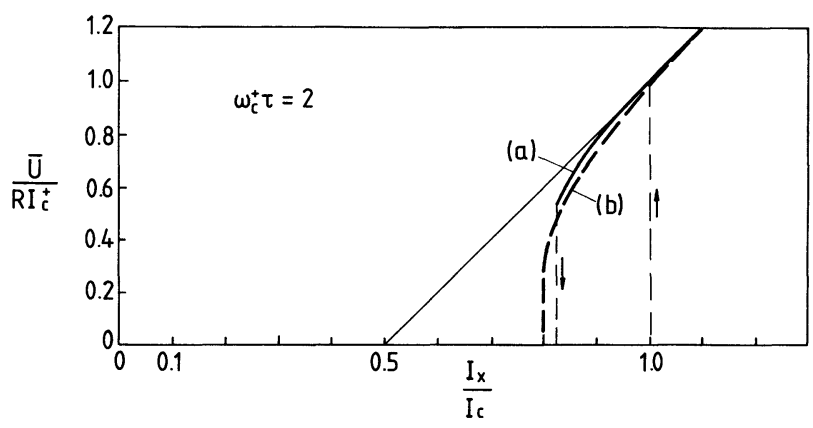

Fig. 7. - (a) I-U-characteristic for the triangular CPR of figure $6 a$. (b) I-U-characteristic for the modified sinusoidal CPR of figure $6 b$. $\omega_{\mathrm{c}}^{+} \tau=2$ (see ref. [15]).

In comparison the $I-U$-characteristic is shown for the triangular CPR as given in figure $6 a[15]$. Obviously with regard to the $I-U$-characteristic the modified sinusoidal CPR is a satisfying approximation of the triangular CPR.

According to [16] the hysteresis-parameter

$$
\alpha_{1}^{+}=I_{1}^{+} / I_{\mathrm{c}}^{+}\left(I_{1}^{+}=I_{1}-I_{2}\right)
$$

is related to $\omega_{\mathrm{c}}^{+} \tau$ by

$$
\left(\omega_{\mathrm{c}}^{+} \tau\right)^{2}=\left[2-(\pi-2) \alpha_{1}^{+}\right] /\left(\alpha_{1}^{+}\right)^{2} .
$$

Therefore it is also possible in case of triangular CPR to determine the effective capacitance $C$ from the measured values of $I_{\mathrm{c}}^{+}, I_{1}^{+}$and $R$. From (11) and (14) one immediately obtains

$$
C=\frac{\phi_{0}}{2 \pi} \cdot \frac{1}{R^{2} I_{\mathrm{c}}^{+}} \cdot \frac{2-(\pi-2) \alpha_{1}^{+}}{\left(\alpha_{1}^{+}\right)^{2}} .
$$

For the evaluation the concept of the modified sinusoidal CPR is used. For the two different states of the contact according to figure $3 \alpha$ and figure $3 \beta$ respectively one yields :
( $\alpha) \alpha_{1}^{+}=0.10$
(B) $\alpha_{1}^{+}=0.125$
$C \simeq 11 \mathrm{pF}$
$C \simeq 12.5 \mathrm{pF}$. 
These values are much higher than the estimated values for the geometric capacitance of point contacts and microbridges. In [17] there is given a plausible explanation for this fact.

3.2 Flux Penetration. - The external magnetic flux $\phi_{x}$ causes the countercurrent $I$ in the weakly connected sc loop. Then the actual flux $\phi$ threading the loop is given by

$$
\phi=\phi_{x}-L I,
$$

where $L$ is the inductance of the loop. $L$ depends on the geometry of the loop only and is not affected by the properties of the contact.

Combining fluxoid quantization condition

$$
\delta=2 \pi \frac{\phi}{\phi_{0}}+2 \pi n ; \quad n \in Z,
$$

the Josephson-relation (4) and eqs. (1), (2) one obtains

$$
\begin{gathered}
\frac{\mathrm{d}^{2} \varphi}{\mathrm{d} t_{1}^{2}}+\beta \frac{\mathrm{d} \varphi}{\mathrm{d} t_{1}}+\varphi+\Gamma f(\varphi)=\varphi_{x} \\
\left(\Gamma=\frac{L I_{\mathrm{c}}}{\phi_{0}}, \quad \beta=\frac{\sqrt{L C}}{R C}\right. \\
\left.t_{1}=\frac{1}{\sqrt{L C}} t, \quad \varphi_{(x)}=\frac{\phi_{(x)}}{\phi_{0}}\right) .
\end{gathered}
$$

This differential equation describes the dynamics of flux penetration within the frame of the RSJ-model. For relevant CPRs (Fig. 5, Fig. 6), however, (18) cannot be solved analytically.

Therefore the solution $\varphi\left(t_{1}\right)$ was calculated by digital [5] and analog [18] computing methods, but only the case of the sinusoidal CPR was treated. For small values of $\Gamma(\Gamma<10)$ the analog computer calculations have been extended to the case of triangular CPRs using a new and simple technique to simulate the nonlinear part $\Gamma f(\delta)$ in (18) [19]. In case of the sinusoidal CPR as well as in case of triangular CPRs one obtains a typical pulse for $\mathrm{d} \varphi / \mathrm{d} t_{1}$, whose shape is characterized by a short increasing time $\left(\simeq 10^{-12} \mathrm{~s}\right)$ well established oscillations on top (about $10^{12} \mathrm{~Hz}$, see ref. [5]) and a sudden breakdown. The number of the oscillations is determined by the number of the flux quanta $\phi_{\mathrm{E}} / \phi_{0}$ penetrating into the loop.

In our experiments (time resolution $\approx 10^{-9} \mathrm{~s}$ ) we cannot observe the oscillations on top of the voltage pulse (see Fig. 4). Therefore we have done a simple calculation which leads to the typical pulse for $\mathrm{d} \varphi / \mathrm{d} t_{1}$, but averages through the oscillations.

For the following considerations we always assume $\Gamma=L I_{\mathrm{c}} / \phi_{0} \gg 1\left(\Gamma>10^{4}\right.$ in our experiments). Then the countercurrent $I$ increases linearly with the external flux $\phi_{x}$ as long as $\phi_{x}$ is smaller than the critical value $\phi_{x \mathrm{c}} \simeq L I_{\mathrm{c}}=\Gamma \phi_{0}$. If $\phi_{x}$ exceeds $\phi_{x \mathrm{c}}$, $I$ exceeds the critical current $I_{\mathrm{c}}$ of the contact and according to the $I-U$-characteristic a voltage $\bar{U}=R I_{\mathrm{c}}^{+}$ appears across the contact (transition A-A', Fig. 8). The suddenly arising voltage causes the start of a flux quantum transition. When the flux penetrates into the loop, the circulating current in the loop starts to decrease. The exact time dependence of $I$ during the flux penetration can only be given, if the solution of the differential eq. (18) is known. When $I$ is down to $I_{1}$, the voltage across the contact vanishes and the flux penetration is terminated (transition $\mathrm{B}^{\prime}-\mathrm{B}$, Fig. 8). Now the current $I$ increases again with external flux $\phi_{x}$ until $I$ reaches $I_{c}$, and the next flux quantum transition starts.

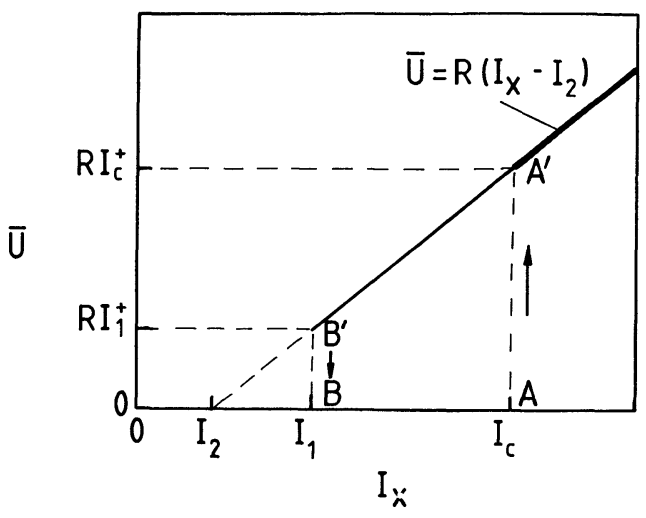

a)

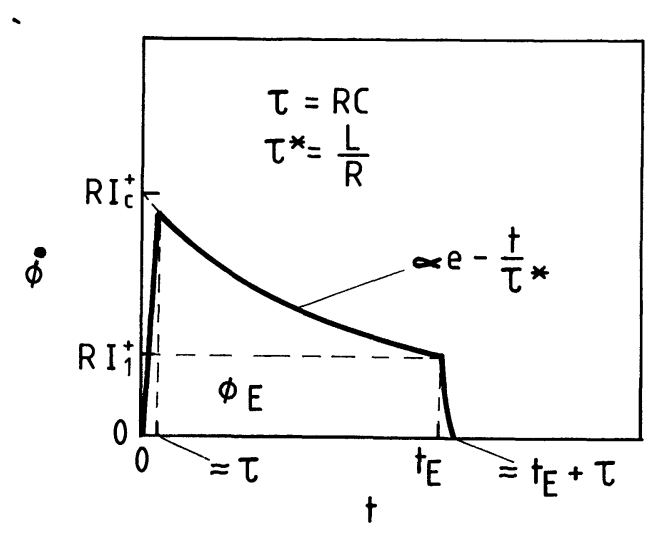

b)

Fig. 8. - Schematical representation of measured $I-U$-characteristic $(a)$ and voltage puls due to flux penetration $(b)(\tau=R C$, $\left.\tau^{*}=L / R\right)$

The flux $\phi_{\mathrm{E}}$ penetrating into the loop during one flux quantum transition is given by

$$
\phi_{\mathrm{E}}=L\left(I_{\mathrm{c}}-I_{1}\right)=L\left(I_{\mathrm{c}}^{+}-I_{1}^{+}\right) .
$$

Using the definitions for the hysteresis-parameter $\alpha_{1}=I_{1} / I_{\mathrm{c}}$ and (13) respectively, eq. (19a) turns into

$$
\varphi_{\mathrm{E}}=\frac{\phi_{\mathrm{E}}}{\phi_{0}}=\Gamma\left(1-\alpha_{1}\right)=\Gamma^{+}\left(1-\alpha_{1}^{+}\right)
$$


with

$$
\Gamma^{+}=\frac{L I_{\mathrm{c}}^{+}}{\phi_{0}} .
$$

For the calculation mentioned above we have made the following assumptions :

a) $\varphi_{x}$ varies quasistatically in time, i.e.

$$
\varphi_{x}=\varphi_{x \mathrm{c}}=\Gamma=\mathrm{constant}
$$

during a flux quantum transition $A-A^{\prime}-B^{\prime}-B$ (see Fig. 8).

b) During a flux quantum transition the exact value of the supercurrent $I_{\mathrm{s}}\left(t_{1}\right)$ can be replaced by its time average value $\left(I_{\mathrm{s}} \approx I_{2}\right.$ for a modified sinusoidal CPR).

c) During the transitions A-A $: I_{\mathrm{s}}=I_{\mathrm{c}} \rightarrow I_{\mathrm{s}}=I_{2}$ and $\mathrm{B}^{\prime}-\mathrm{B}: I_{\mathrm{s}}=I_{2} \rightarrow I_{\mathrm{s}}=I_{1}$ the supercurrent changes its value infinitely fast, i.e. the relaxation time for the supercurrent is zero.

From (18) we therefore obtain the differential equations

$$
\begin{gathered}
\frac{\mathrm{d}^{2} \varphi}{\mathrm{d} t_{1}^{2}}+\beta \frac{\mathrm{d} \varphi}{\mathrm{d} t_{1}}+\varphi=\varphi_{x \mathrm{c}}-\frac{L I_{2}}{\phi_{0}}=\Gamma^{+} \\
\varphi(0)=0, \quad\left(\frac{\mathrm{d} \varphi}{\mathrm{d} t_{1}}\right)_{\mathrm{I}_{1}=0}=0
\end{gathered}
$$

(branch A-A'-B')

$$
\begin{aligned}
& \frac{\mathrm{d}^{2} \varphi}{\mathrm{d} t_{1}^{2}}+\beta \frac{\mathrm{d} \varphi}{\mathrm{d} t_{1}}=0 \\
&\left(\frac{\mathrm{d} \varphi}{\mathrm{d} t_{1}}\right)_{t_{1}=\frac{1}{\sqrt{L C}} I_{\mathrm{E}}}=\frac{1}{\beta} \frac{L I_{1}^{+}}{\phi_{0}}
\end{aligned}
$$

(transition B'-B)

for the nonequilibrium state of the contact and

$$
\varphi=\varphi_{x}-\frac{L I_{\mathrm{s}}}{\phi_{0}}
$$

(branch B-A; especially

$$
\left.\varphi_{\mathrm{E}}=\varphi_{x \mathrm{c}}-\frac{L I_{1}}{\phi_{0}}=\Gamma^{+}\left(1-\alpha_{1}^{+}\right) \text {at B }\right)
$$

for the equilibrium state of the contact.

For $\beta \gg 2$ (valid for all experiments in this work) the solution of $(20)$ is

with

$$
\varphi(t)=\frac{\phi(t)}{\phi_{0}}=\Gamma^{+}[1-A(t)]
$$

$$
\begin{aligned}
A(t) & =\frac{1}{1-\frac{2}{\beta^{2}}}\left[\left(1-\frac{1}{\beta^{2}}\right) \mathrm{e}^{-t / \tau^{*}}-\frac{1}{\beta^{2}} \mathrm{e}^{-t / \tau}\right] \\
\tau^{*} & =L / R, \quad \tau=R C .
\end{aligned}
$$

Comparing eqs. (23) and (19b) we immediately obtain the termination condition for the flux penetration

$$
A\left(t_{\mathrm{E}}\right)=\alpha_{1}^{+} .
$$

Furthermore we yield an analytical expression for the voltage pulse :

$\dot{\phi}(t)=\left\{\begin{array}{cc}R I_{\mathrm{c}}^{+}\left(\mathrm{e}^{-t / \tau^{*}}-\mathrm{e}^{-t / \tau}\right) ; & 0 \leqslant t \leqslant t_{\mathrm{E}} \\ & \left(\mathrm{A} \rightarrow \mathrm{A}^{\prime} \rightarrow \mathrm{B}^{\prime}\right) \\ R I_{1}^{+} \exp \left(-\frac{t-t_{\mathrm{E}}}{\tau}\right) ; & t \geqslant t_{\mathrm{E}} \\ \left(\mathrm{B}^{\prime} \rightarrow \mathrm{B}\right)\end{array}\right.$.

The graphical representation (Fig. 8b) demonstrates the essential properties of the voltage pulse for the case

$$
\beta=\sqrt{\frac{\tau^{*}}{\tau}} \gg 2:
$$

short increasing time $\tau\left(\tau \approx 10^{-12} \mathrm{~s}\right)$, smooth decay for $\tau<t \leqslant t_{\mathrm{E}}$ and sudden breakdown $(\approx \tau)$ thereafter.

In addition, from (26) the mean penetration frequency for the flux

$$
v_{\mathrm{M}}=\frac{\phi_{\mathrm{E}}}{\phi_{0}} \cdot \frac{1}{t_{\mathrm{E}}}
$$

can be determined. For $t=t_{\mathrm{E}} \gg \tau$ we get the condition : $R I_{1}^{+}=R I_{\mathrm{c}}^{+} \mathrm{e}^{-t_{\mathrm{E}} / \tau^{*}}$. This leads to

$$
v_{\mathrm{M}}=-\frac{R I_{\mathrm{c}}^{+}}{\phi_{0}} \frac{1-\alpha_{1}^{+}}{\ln \alpha_{1}^{+}} \text {. }
$$

The analytical expression for the voltage pulse (26) allows one to find out the time constant $\tau^{*}$ from

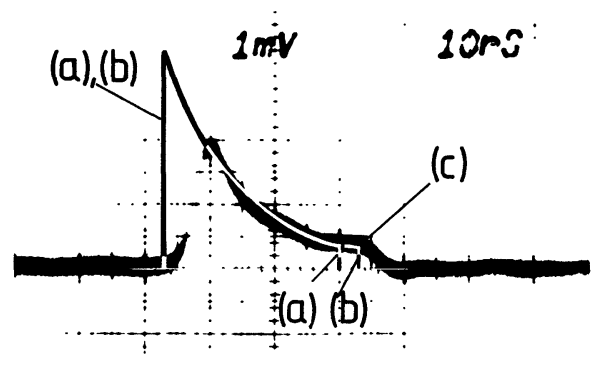

(x)

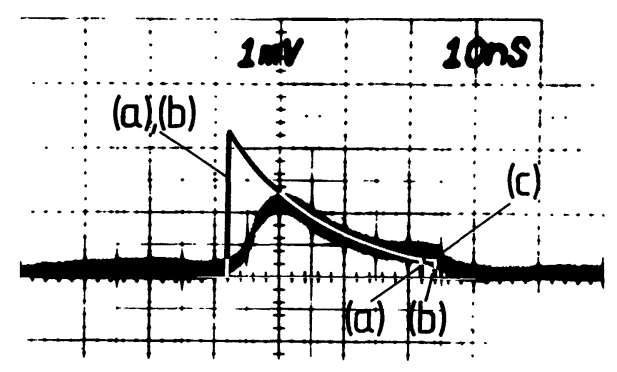

( $\beta)$

Fig. 9. - Comparison of calculated $(a)$, corrected $(b)$ and measured (c) voltage pulses due to flux penetration for case $\alpha$ and $\beta$ respectively. 
the smooth decaying branch of the measured pulse (Fig. $4 \alpha, \beta$ ). With the value for $R$ taken from the corresponding $I-U$-characteristic (Fig. $3 \alpha, \beta$ ) one always obtains $L=(20 \pm 2) \mathrm{nH}$ for the inductance of the experimental set-up used here. Furthermore, taking the values for the parameters $I_{\mathrm{c}}^{+}, I_{1}^{+}$and $R$ from the $I-U$-characteristic, $\dot{\phi}(t)$ can be predicted according to (26).

One obtains the calculated pulse (Fig. 9a).

Because of the experimental technique used the measured pulse (Fig. 9c) has to be corrected. As the bandwidth of the scope used is limited $(100 \mathrm{MHz})$ the recording cannot follow the increasing process with its time constant $\tau \approx 10^{-12} \mathrm{~s}$, but reaches the decaying branch after $10^{-8} \mathrm{~s}$. This is the reason why the shape of the pulse and so especially the area under the pulse ( $\hat{=}$ penetrated flux) is not correctly displayed by the scope. So, by back-extrapolation of the decaying branch and an instant step at $t=0$ on the time scale of the measured pulse we determine the real shape of the increasing process. The breakdown of the pulse has to. be treated in the same way. This procedure leads to the corrected pulse (Fig. 9b) from which we take the data for the penetrated flux $\phi_{\mathrm{E}}$ listed below.

Comparing the corrected pulse and the calculated pulse predicted by the $I-U$-characteristic only one obtains an excellent agreement in respect to the shape of the voltage pulse, the amount of the penetrated flux and the mean penetration frequency.
Case $\alpha$

$\begin{array}{lc}\phi_{\mathrm{E}}[\mathrm{Vs}] & v_{\mathrm{M}}[\mathrm{Hz}] \\ 36 \times 10^{-12} & 0.64 \times 10^{12} \\ 1) \times 10^{-12} & 0.60 \times 10^{12}\end{array}$

Case $\beta$

$\begin{array}{cc}\phi_{\mathrm{E}}[\mathrm{Vs}] & v_{\mathrm{M}}[\mathrm{Hz}] \\ - & - \\ 28 \times 10^{-12} & 0.46 \times 10^{12} \\ (29 \pm 1) \times 10^{-12} & 0.44 \times 10^{12}\end{array}$

4. Correlation between flux quantum transition and I-U-characteristic. - The correlation between flux quantum transition and $I-U$-characteristic is clearly demonstrated by eq. (19) :

For $\alpha_{1}=1$ or $\alpha_{1} \leqslant 1$ (that means almost no hysteresis) only single flux quantum transitions may occur. But a well established hysteresis $\left(\alpha_{1}<1\right)$ always leads to multiple flux quantum transitions.

In the case of sinusoidal and modified sinusoidal CPRs we have

$\beta=\sqrt{2 \pi \Gamma^{+}}\left[\frac{\left(\alpha_{1}^{+}\right)^{2}}{2-(\pi-2) \alpha_{1}^{+}}\right]^{1 / 2} \equiv \beta\left(\alpha_{1}^{+}\right)$.

Let us define

$\beta_{\mathrm{c}}=\beta\left(\alpha_{1}=1\right)=\sqrt{\frac{2 \pi}{4-\pi}} \sqrt{\Gamma}=2.71 \sqrt{\Gamma}$;

then we obtain an universal relationship between the parameter $\alpha_{1}$ which describes the hysteresis of the $I-U$-characteristic and the parameter $\beta / \beta_{\mathrm{c}}$ which describes the type of the flux quantum transition :

$$
\frac{\beta}{\beta_{\mathrm{c}}}=\sqrt{4-\pi} \sqrt{\frac{I_{\mathrm{c}}^{+}}{I_{\mathrm{c}}}}\left[\frac{\left(\alpha_{1}^{+}\right)^{2}}{2-(\pi-2) \alpha_{1}^{+}}\right]^{1 / 2} .
$$

Note that the ratio $\beta / \beta_{\mathrm{c}}$ is independent of $L$.

$$
\begin{gathered}
\text { I-U-characteristic } \\
- \\
\alpha_{1} \lesssim 1
\end{gathered}
$$

(weak hysteresis)

$$
\alpha_{1}<1
$$

(strong hysteresis)
Flux quantum transition

$$
\frac{\beta}{\beta_{\mathrm{c}}} \lesssim 1
$$

(single flux quantum transition)

$$
\frac{\beta}{\beta_{\mathrm{c}}}<1
$$

(multiple flux quantum transition)

Combining (19) and (30) we get an universal function

$\frac{1}{\Gamma} \frac{\phi_{\mathrm{t}}}{\phi_{0}}=\frac{I_{\mathrm{c}}^{+}}{I_{\mathrm{c}}}\left\{1-\frac{x}{2(4-\pi)}\left[-(\pi-2) x+\sqrt{(\pi-2)^{2} x^{2}+8(4-\pi)}\right]\right\} \quad$ with $\quad x=\sqrt{\frac{I_{\mathrm{c}}}{I_{\mathrm{c}}^{+}}} \frac{\beta}{\beta_{\mathrm{c}}}$.

Figure 10 shows the plot of (31) for the sinusoidal CPR $(b)$ and for modified sinusoidal CPRs $(c)-(e)$ in comparison with curve (a) numerically calculated by [5] for the sinusoidal CPR. There is an excellent agreement between the curves $(a)$ and $(b)$. All the plots $(b)-(e)$ are obtained from the correlation between flux quantum transition and $I-U_{1}$-characteristic in the frame of the RSJ-model.
It seems that the curves $\frac{1}{\Gamma} \frac{\phi_{\mathrm{E}}}{\phi_{0}} v s . \frac{\beta}{\beta_{\mathrm{c}}}$ depend on the type of the CPR in a sensitive way as shown here for the sinusoidal and modified sinusoidal CPRs. The experimental points $(+)$ taken from the corrected pulses figures $9 \alpha(b), 9 \beta(b)$ lie almost exactly on the corresponding curves $(c)$ and $(d)$. This is due to the 
excellent agreement of calculated and corrected pulses (Fig. 9).

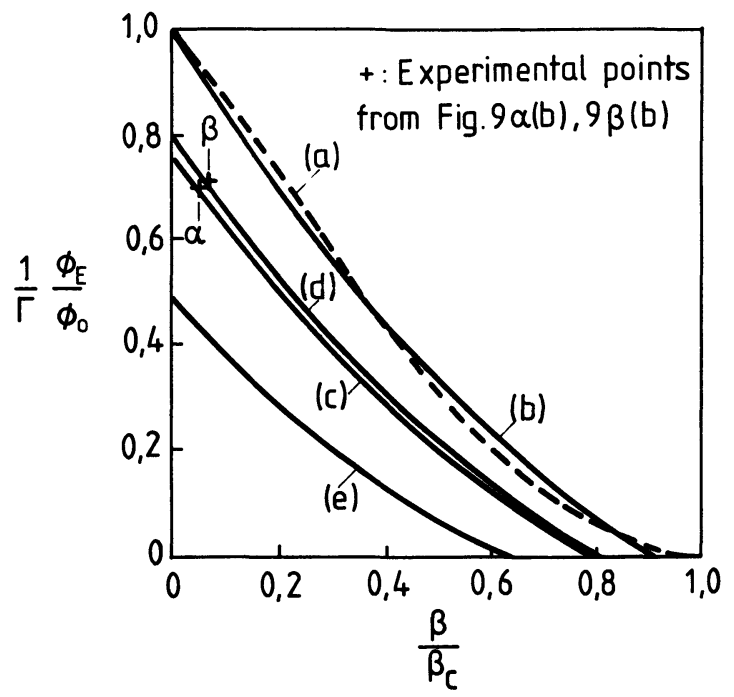

Fig. 10. - Universal curve which describes the dependence of entering flux $1 / \Gamma \phi_{\mathrm{E}} / \phi_{0}$ on the parameter $\beta / \beta_{\mathrm{c}}\left(\beta_{\mathrm{c}}=2.97 \sqrt{\Gamma}\right)$. (a) From [5], sinusoidal CPR $\left(\beta_{\mathrm{c}}=2.97 \sqrt{\Gamma}\right)$. (b)-(e) This paper, plot of eq. (31) $\left(\beta_{\mathrm{c}}=2.71 \sqrt{\Gamma}\right)$. (b) Sinusoidal CPR : $I_{\mathrm{c}}^{+}=I_{\mathrm{c}}$ modified sinusoidal CPR : (c) $I_{\mathrm{c}}^{+}=0.77 I_{\mathrm{c}}(\operatorname{case} \alpha),(d) I_{\mathrm{c}}^{+}=0.80 I_{\mathrm{c}}$ (case $\beta),(e) I_{\mathrm{c}}^{+}=0.50 I_{\mathrm{c}}$.
5. Discussion. - The rather simple RSJ-model describes the very complex phenomena, $I-U$-characteristic and flux penetration into a weakly connected sc loop, surprisingly well. Comparison of measurements of the $I-U$-characteristic and of flux penetration leads to an excellent agreement.

Since the prediction of the voltage pulse for a large number of penetrating flux quanta cannot be treated analytically in the full formalism of the RSJ-model, a simple approximation was made that still contains the essentials of the RSJ-model.

The CPR does not enter into this approximation explicitly, but only the CPR justifies the introduction of the termination condition for the entering flux and the back-jump into the sc state at $I=I_{1}$.

In the same way $I_{2} \neq 0$ is understood as a direct result of a reentrant CPR.

The full CPR cannot be determined by these experiments, but this does not diminish the essential importance of the CPR for the considerations carried out here.

In addition to the estimation $v_{\mathrm{M}}=R I_{\mathrm{c}}^{+} / \phi_{0}$ given by [5] we have a factor $\left(1-\alpha_{1}^{+}\right) / \ln \alpha_{1}^{+}$in $(27)\left(\approx \frac{1}{3}\right.$ for this work) which describes our experiments very well. It needs further effort to examine experimentally such a dependence of $v_{M}$ on the CPR.

\section{References}

[1] Silver, A. H., Zimmerman, J. E., Phys. Rev. 157 (1967) 317.

[2] See for instance : Proceedings of the International Conference on Superconducting Quantum Devices, Berlin (West), (1976); Ed. H. D. Hahlbohm, H. Lübbig, Walter de Gruyter (Berlin, New York) 1977.

[3] Kurkijärvi, J., Phys. Rev. B 6 (1972) 832.

[4] Sullivan, D. B. et al., J. Appl. Phys. 41 (1970) 4865. Sandell, R. D. et al., J. Appl. Phys. 44 (1973) 3312.

[5] Smith, H. J. T., Blackburn, J. A., Phys. Rev. B 12 (1975) 940.

[6] Stewart, W. C., Appl. Phys. Lett. 12 (1968) 277. McCumber, D. E., J. Appl. Phys. 39 (1968) 3113.

[7] Skocpol, W. J., Beasley, M. R., Tinkham, M. - J. Appl. Phys. 45 (1974) 4054 ; J. Low Temp. Phys. 16 (1974) 145.
[8] A phenomenological discussion of the effects arising in the resistive superconducting state is given by MERCEREAU, J. E., Rev. Phys. Appl. 5 (1970) 13.

[9] Bloch, F., Phys. Rev. B 2 (1970) 109.

[10] Barnes, L. J., Phys. Rev. 184 (1969) 434.

[11] Aslamazov, L. G., LaRkin, A. I., JETP Lett. 9 (1969) 87.

[12] Baratoff, A. et al., Phys. Rev. Lett. 25 (1970) 1096, E 1738.

[13] Blackburn, J. A. et al., J. Low Temp. Phys. 20 (1975) 523.

[14] Gregers-Hansen, P. E. et al., J. Low Temp. Phys. 7 (1972) 99.

[15] Auracher, F., van Duzer, T., Appl. Phys. Lett. 21 (1972) 515.

[16] ZAPPe, H. H., J. Appl. Phys. 44 (1973) 1371.

[17] Song, Y., J. Appl. Phys. 47 (1976) 2651.

[18] ERNÉ, S. N., LUTHER, H., PTB Berlin, private communication.

[19] Voss, G., Hausen, A., To be published. 